\title{
Multiple system atrophy: natural history, MRI morphology, and dopamine receptor imaging with ${ }^{123}$ IBZM-SPECT
}

\author{
J B Schulz, T Klockgether, D Petersen, M Jauch, W Müller-Schauenburg, S Spieker, \\ K Voigt, J Dichgans
}

\begin{abstract}
Sixteen patients with a clinical diagnosis of probable multiple system atrophy (MSA) were examined clinically by MRI and by ${ }^{123}$ I-iodobenzamide single photon emission computed tomography (IBZMSPECT). The clinical records of another 16 patients were also analysed retrospectively. On the basis of their clinical presentation, patients were subdivided into those with prominent parkinsonism (MSA-P, $n=11$ ) and those with prominent cerebellar ataxia (MSA-C, $n=21$ ). Autonomic symptoms were present in all patients and preceded the onset of motor symptoms in $63 \%$ of patients. Calculated median lifetime and the median time to become wheelchair bound after onset of disease were significantly shorter for MSA-P than for MSA-C (lifetime: 4.0 $v$ 9.1 years; wheelchair: $3 \cdot 1 v 5.0$ years) suggesting a better prognosis for cerebellar patients. A significant loss of striatal dopamine receptors (below 2 SD threshold) was detected by IBZM-SPECT in $63 \%$ of the patients $(56 \%$ below $2 \cdot 5$ SD threshold). There was no difference between patients with MSA-C and those with MSA-P in the proportion with significant receptor loss and the extent of dopamine receptor loss. Planimetric MRI evaluation showed cerebellar and brainstem atrophy in both groups. Atrophy was more pronounced in patients with MSA-C than in those with MSA-P. Pontocerebellar hyperintensities and putaminal hypointensities on $T 2$ weighted MRI were found in both groups. Pontocerebellar signal abnormalities were more pronounced in MSA-C than in MSA-P, whereas the rating scores for area but not for intensity of putaminal abnormalities were higher in MSA-P. MRI and IBZM-SPECT provide in vivo evidence for combined basal ganglia and pontocerebellar involvement in almost all patients in this series.
\end{abstract}

(F Neurol Neurosurg Psychiatry 1994;57:1047-1056)

The term multiple system atrophy (MSA), first used by Graham and Oppenheimer in 1969,1 refers to a sporadically occurring adult onset neurodegenerative disease. Neuropathologically, MSA is characterised by the presence of olivopontocerebellar atrophy, striatonigral degeneration, and degeneration of the intermediolateral cell columns of the spinal cord. All of these changes are not necessarily found in each patient with MSA. In some patients, degeneration affects mainly the basal ganglia, whereas in others the pontocerebellar system or the spinal cord are more affected. Therefore, evidence of cell degeneration and gliosis in at least three of the following anatomical structures is thought to be sufficient for the neuropathological diagnosis of MSA: striatum, substantia nigra, Purkinje cell layer of the cerebellar cortex, pontine nuclei, inferior olives, and intermediolateral cell columns of the spinal cord. ${ }^{2-4}$ Striatum or substantia nigra must be one of these areas. ${ }^{5}$ By contrast with idiopathic Parkinson's syndrome, there is no Lewy body pathology in MSA. Rather, a recent study had reported the presence of glial cytoplasmatic inclusion bodies in MSA. ${ }^{6}$ The specificity of this finding remains to be proved in further studies.

The clinical presentation of patients with MSA is highly variable, at least at the onset of the disorder: patients may present with parkinsonism, with a cerebellar syndrome, with progressive autonomic failure, or with variable combinations of these syndromes. Usually parkinsonian symptoms respond only poorly to levodopa. Only a minority of patients with MSA are correctly diagnosed during life due to equivocal clinical symptomatology and the lack of definite diagnostic tests. Accordingly, some patients with MSA are simply diagnosed as having Parkinson's disease. Other patients are categorised under the umbrella terms Parkinson-plus syndrome or atypical parkinsonism. Patients with a combination of parkinsonism and autonomic failure are often referred to as having Shy-Drager syndrome. On the other hand, MSA may be the underlying pathology in patients with prominent cerebellar ataxia who received the diagnosis of sporadic olivopontocerebellar atrophy or idiopathic cerebellar ataxia during life. In an attempt to form a rational basis for the intra vitam diagnosis of MSA, Quinn ${ }^{7}$ proposed clinical criteria for the diagnosis of probable MSA; these include the presence of parkinsonism or cerebellar ataxia and severe symptomatic autonomic failure without dementia and downgaze palsy. Dependent on whether parkinsonian or cerebellar symptoms predominate the diagnosis is parkinsonian (MSA-P) or cerebellar (MSA-C) (probable) MSA.

In recent years, modern imaging methods such as MRI, ${ }^{8-11}$ SPECT, ${ }^{12}{ }^{13}$ and PET ${ }^{14}{ }^{15}$ have increasingly been used in neurodegenera- 
tive diseases. Although these methods have also been applied to patients with MSA, their diagnostic value in MSA remains uncertain. The reported abnormalities, such as putaminal iron accumulation or loss of dopamine receptors, are found in other parkinsonian syndromes. Also, most studies are restricted to patients with MSA who have parkinsonian symptomatology, whereas patients with cerebellar symptoms have attracted much less attention.

In this study, we report clinical, MRI, and ${ }^{123}$ I-iodobenzamide (IBZM) single photon SPECT data from a series of 16 patients with probable MSA of either parkinsonian (MSAP) or cerebellar (MSA-C) type. The aim of the present study was twofold. Firstly, we wished to study the natural history and prognosis of MSA and to compare MSA-C and MSA-P. Secondly, we wished to study the abnormalities detected by MRI and IBZMSPECT in patients with MSA. In particular, we were interested in whether these imaging procedures can identify abnormalities that are common to all patients with MSA or whether they yield different results in cerebellar and parkinsonian patients.

\section{Patients and methods \\ PATIENTS}

The clinical records of all patients with parkinsonian symptoms $(n=850)$ and all patients with cerebellar degenerative disease ( $n=217$ ) referred to our department between 1983 and 1991 were reviewed. Of these, 32 fulfilled all of the following four diagnostic criteria for probable MSA: (a) progressive cerebellar ataxia and/or non-responsive or poorly responsive levodopa parkinsonism without evidence for a focal or non-focal symptomatic origin of the disorder; $(b)$ severe symptomatic autonomic failure with at least postural syncope or presyncope and/or pronounced urinary incontinence or retention not due to other causes; (c) absence of any neurodegenerative disorders in relatives and no evidence of consanguinity of parents; (d) absence of dementia according to DSM-III $\mathrm{R}$ criteria, generalised tendon areflexia, or predominant downgaze supranuclear palsy. Based on their clinical presentation, patients were subdivided into two groups, one with predominant cerebellar symptoms (MSA-C, $\mathbf{n}=21$ ) and another with predominant parkinsonian presentation (MSA-P, $\mathrm{n}=11$ ).

16 patients were personally interviewed and clinically examined by one of us (JBS) with a standardised examination procedure. Their mean age was $60 \cdot 1$ (SD 8.3) years. Severity of cerebellar symptoms was rated on a scale ranging from 0 (absent) to 5 (most severe). ${ }^{16}$ We also used the Hoehn and Yahr scale ${ }^{17}$ and the unified Parkinson's disease rating scale (UPDRS) ${ }^{18}$ for rating the severity of parkinsonian patients, and the Kurtzke EDSS scale ${ }^{19}$ for estimation of global disability state. Clinical examination included measurement of blood pressure and heart rate in the supine position and one and five minutes after active standing. Orthostatic reduction in systolic blood pressure of $30 \mathrm{~mm} \mathrm{Hg}$ or more without a sufficient increase of heart rate was considered orthostatic hypotension. On the day of the clinical investigation MRI and IBZMSPECT were performed. Two patients in whom MRI could not be performed due to claustrophobia or a metallic implant received a high resolution cranial CT examination.

Those patients with probable MSA who were not seen personally $(n=16)$ were included only in the outcome evaluation. For these patients, age at onset of first notable motor symptom, clinical type (parkinsonian $v$ cerebellar), duration after onset of motor symptoms until they became wheelchair bound, and age at death were determined from the clinical records. In many patients autonomic symptoms antedated motor symptoms by several years. Autonomic symptoms were often vague and of undetermined onset, however, so that it turned out to be impossible to use them for exact temporal definition of onset of disease in the 16 patients studied retrospectively.

MRI

MRI was performed with a $1.5 \mathrm{~T}$ superconducting system (Magnetom, Siemens AG, Erlangen, Germany) with a circularly polarised head coil. Data were displayed on a $256 \times 256$ matrix. All patients were investigated by a standard examination programme that included sagittal and axial $\mathrm{T} 1$ weighted images (spin echo, TR $=600 \mathrm{~ms}$, $\mathrm{TE}=15$ $\mathrm{ms}$, slice thickness $=4 \mathrm{~mm}$ ), and axial proton-density and T2 weighted images (dual spin echo, $T R=2100 \mathrm{~ms}, \mathrm{TE}=45+90$ $\mathrm{ms})$. To improve slice profiles two subsequent sets of measurements of each sequence were made, each with an interslice gap of $4 \mathrm{~mm}$. Both sets of measurements together gave a complete series of images without a gap. Special care was taken to position the head and neck symmetrically in the head coil. Twenty-four age and sex matched healthy persons served as controls (mean age 58.0 $(15 \cdot 6)$ years). Neurological examination of these persons was normal and routine evaluation of their MRIs gave no pathological findings.

Quantitative MRI evaluation of cerebellum, pons, brainstem, and spinal cord was performed under blinded conditions. Regions of interest of any form, location, and size could be outlined with a computer program (fig 3). For quantitative planimetric evaluation of areas we used an image analyser and a computerised interpretation program..$^{20} 21$ This technique permits measurements of the amount of tissue within a region of interest without lining out the border between tissue and CSF by hand. Furthermore, it takes into account partial volume effects that often occur at the surface of the cerebellum. For each case the individual signal intensities of CSF and of the cerebellar tissue were defined. Those measurements gave two sets of normal distributed brain tissue and CSF grey values. Pixels with values equal or higher than the 
mean tissue value were counted as tissue, whereas pixels with values equal or lower than the mean CSF value were counted as CSF. The remaining pixels were designated as containing both tissue and CSF. It was assumed that the difference in signal intensity between grey and white matter could be ignored for this purpose. Those voxels with signal intensities between the mean values of brain tissue and CSF were linearly weighted according to their tissue content. This allowed us to finally summarise the number of effective tissue pixels and to obtain corrected planimetric values for cerebellar structures (see fig 3). To compensate for individual variations in head size we measured the total area of the posterior fossa on the midsagittal slice and related each planimetric measurement to the respective posterior fossa area. There were no statistically significant differences in the area of the posterior fossa between controls and patients with MSA (mean (SD); controls 4922 (379) pixels; MSA-P 4910 (445) pixels; MSA-C: 4834 (480) pixels).

The areas of the cerebellar vermis and the basis pontis were measured on the midsagittal slice. As we obtained a complete series of images without a gap, the midsagittal slice was easily identifiable by showing the cerebral aquaeduct in its complete craniocaudal extent. The basis pontis was delineated as an elliptical structure: the ventral border was obvious and the dorsal border was determined by the medial lemniscus, which appeared as a narrow strip of reduced signal intensity. The areas of the cerebellar hemispheres were calculated on the first parasagittal slice lateral to the lateral border of the middle cerebellar peduncle. As there were no systematic size differences between the hemispheres of the two sides, we calculated the mean of both sides for further analysis. We also measured the area of medulla oblongata in the horizontal plane at the level of the inferior olivary complex and the cervical spinal cord on the horizontal plane at the level of the dens. Also the diameter $(\mathrm{mm})$ of the middle cerebellar peduncle was measured in a horizontal plane with a distance algorithm.

The degree of cortical atrophy and the severity of signal abnormalities on T2 weighted images were rated independently by two of us (JBS, DP). To ensure uniform and anonymous quality of the images, all images were documented on $x$ ray films without any identification data. The degree of cortical atrophy and hypointensities of the putamen, pallidum, substantia nigra, and red and cerebellar dentate nucleus were rated on a scale ranging from 0 to 3 . Grade 0 indicated complete absence, grade 1 mild, grade 2 moderate, and grade 3 strong atrophy or hypointensity respectively. The area of signal hypointensity in the putamen was also rated. Grade 0 indicated no hypointensity, grade 1 hypointensity limited to the lateral margin of the putamen, grade 2 hypointensity extending through part of the body of the putamen, and grade 3 hypointensity throughout the entire putamen. ${ }^{11}$ Hyperintensities of the pons and middle cerebellar peduncles ${ }^{22}$ were rated to be absent (grade 0), mild (grade 1), moderate (grade 2), or strong (grade 3 ).

\section{SPECT}

IBZM-SPECT was performed two hours after intravenous injection of $185 \mathrm{MBq}{ }^{123} \mathrm{IBZM}$ (3-iodo-6-methoxybenzamide; Cygne BV, Netherlands/Du Pont de Nemour, Germany). If patients were taking medication, this was stopped 24 hours before SPECT scanning. SPECT data were acquired with a dual head rotating gamma Camera (Picker Digital Dyna, AP Collimator) and a PCS 512 computer system (gamma 11). For data acquisition the following standard programme was used: two complete circles $\left(360^{\circ}\right.$ each camera head), 64 frames/circle, $64 \times 64$ matrix, slice thickness $5.5 \mathrm{~mm}$, pixel resolution $5.5 \mathrm{~mm}$, full width at half maximum (FWHM) of the reconstructed line spread function $20 \mathrm{~mm}$. The acquisition time was $40 \mathrm{~s} /$ frame.

We superimposed MRI and SPECT with external landmarks. The orbital meatal line, as shown by a laser system on the SPECT system, was marked by a pencil line and oil filled tubes. This line represents the collimator midline and the first MRI image. After prefiltering the raw data (METZ filter) and overlaying a RAMP filter, SPECT reconstruction was done for axial, coronal, and sagittal slices including attenuation correction with an attenuation coefficient of $0.139 \mathrm{~cm}^{-1}$. Images from MRI $(256 \times 256,16$ bit count depth) were transmitted via ethernet, converted into gamma 11 file format $(256 \times 256,8$ bit count depth), and internally reduced to $128 \times 128$ ( 8 bit count depth) for further procedures. After landmark based slice identification and scale adaptation by zooming, the in plane matching was done under visual control, with a region of interest contour shifting as an intermediate step. To identify the correct anatomical sites axial SPECT and MRI images were superimposed adding then two to three consecutive SPECT images. On these sum images, basal ganglia and prefrontal regions of interest were drawn. The size of the regions of interest varied from patient to patient and ranged from 20 to 30 pixels $\left(6\right.$ to $9 \mathrm{~cm}^{2}$ ). Care was taken that the striatal region of interest, which had an elongated shape, was clearly within the borders of the striatum, as shown by the superimposed MRI, and that the striatal and prefrontal regions of interest of an individual patient had comparable sizes. The prefrontal region of interest had a band-like shape following isocontours. The regions of interest were used for calculating counts per pixel in the basal ganglia and prefrontal cortex. The ratio of these values (BG/FC ratio) then represents a semiquantitative measure of the relative density of basal ganglia dopamine receptors labelled by IBZM. ${ }^{23}$

Because we did not obtain the permission of the local ethics committee to perform IBZM-SPECT in healthy controls, we compared the results of the patients with MSA with a group of untreated de novo patients with idiopathic Parkinson's syndrome (Hoehn 
Figure 1 Survival function $(A)$ and function to become wheelchair bound $(B)$ of patients with $M S A-C$ and $M S A-P$, as calculated by the KaplanMeier life table method. ${ }^{23}$ Squares represent patients with $M S A-P(n=11)$, triangles patients with $\operatorname{MSA}-C(n=21)$. Filled symbols represent completed cases, who died (A) or became wheelchair bound (B); open symbols censored cases, who were lost during follow up or have not died or become wheelchair bound.
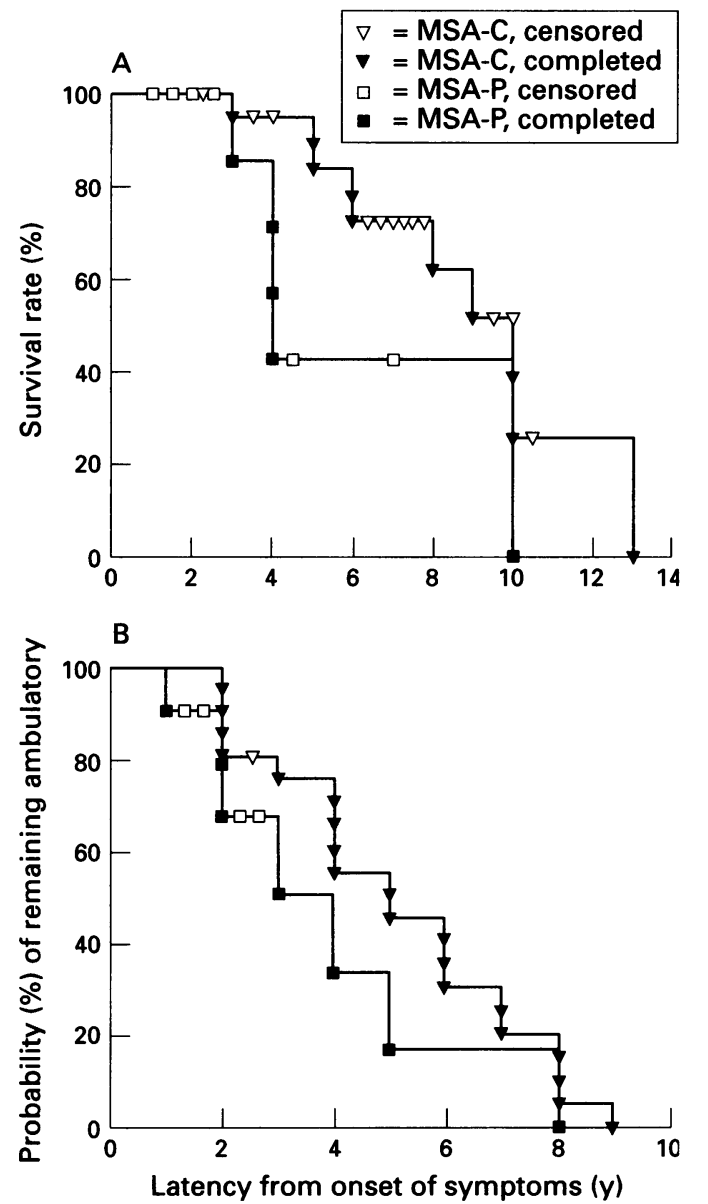

and Yahr stage I and II; mean age $53 \cdot 8(10 \cdot 0)$ years). All patients with idiopathic Parkinson's syndrome underwent an apomorphine test and were subsequently treated with levodopa. Only those patients were included who had a positive apomorphine test and showed pronounced clinical improvement after a three month treatment with levodopa. We believe that it is justified to use levodopa responsive patients with idiopathic Parkinson's syndrome as controls because two earlier studies ${ }^{1213}$ failed to show any differences of the BG/FC uptake ratios of IBZM in healthy persons and untreated patients with idiopathic Parkinson's syndrome. Moreover, the $\mathrm{BG} / \mathrm{FC}$ ratio $(1 \cdot 76(\mathrm{SD} 0 \cdot 1))$ in our idiopathic Parkinson's syndrome group was identical to that reported by Brücke and colleagues $^{12}$ in their healthy controls.

\section{STATISTICAL ANALYSIS}

Parametric variables (age, quantitative morphometric MRI data of infratentorial structures, and IBZM-SPECT data) were statistically analysed by an analysis of variance for each of the dependent variables. The analysis of significant main effects was performed by Duncan's multiple range test. Ordinal variables (MRI rating scores) were analysed by Kruskal-Wallis one way analysis of variance by ranks followed by the MannWhitney $U$ test with Bonferroni adjustment according to the number of tests performed. Survival analysis was performed with the method described by Kaplan and Meier. ${ }^{24}$

\section{Results}

Eleven out of 32 patients with MSA were women and 21 were men. The mean age at the onset of motor symptoms was $54 \cdot 1$ (SD $7 \cdot 1$ ); range 40 to 66 years), with no differences between the subgroups (MSA-C $52 \cdot 8$ years; MSA-P 55.7 years). The calculated median lifetime ${ }^{24}$ after onset of motor symptoms was 8.9 years. It was significantly shorter in MSA-P (4.0 years) than in MSA-C (9.1 years; fig 1A). Similarly, the calculated median time to become wheelchair bound was shorter in MSA-P ( $3 \cdot 1$ years) than in MSA-C ( 5.0 years; fig $1 \mathrm{~B})$.

Tables 1 and 2 give the clinical data of the 16 personally examined patients. The mean duration of motor symptoms was shorter in MSA-P (mean (SD) $3.0(2 \cdot 1)$ years) than in MSA-C (6.6 (2.6); $p<0.05)$. All patients had dysautonomia. The most frequent autonomic symptoms were orthostatic hypotension $(94 \%)$, and urinary incontinence or retention (94\%). All male patients complained of erectile dysfunction. Another common sign was vocal cord dysfunction ( $81 \%$ ) with nocturnal stridor or hoarseness. Three patients required tracheotomy. In one patient a combined cen-

Table 1 Clinical data of personally examined patients

\begin{tabular}{|c|c|c|c|c|c|c|c|c|c|c|c|}
\hline Case & Sex & $\begin{array}{l}\text { Age of } \\
\text { onset } \\
(y)\end{array}$ & $\begin{array}{l}\text { First } \\
\text { symptom }\end{array}$ & $\begin{array}{l}\text { Dur } \\
\text { symp } \\
\text { MO }\end{array}$ & $\begin{array}{l}\text { on of } \\
m s(y) \\
A F\end{array}$ & $\begin{array}{l}\text { Parkinsonian } \\
\text { symptoms }\end{array}$ & $\begin{array}{l}\text { Cerebellar } \\
\text { symptoms }\end{array}$ & $\begin{array}{l}\text { Pyramidal } \\
\text { signs }\end{array}$ & $\begin{array}{l}\text { Kurtzke } \\
\text { score }\end{array}$ & $\begin{array}{l}\text { Levodopa } \\
\text { response }\end{array}$ & $\begin{array}{l}\text { Treatment } \\
\text { at the time of } \\
\text { IBZM-SPECT }\end{array}$ \\
\hline \multicolumn{12}{|c|}{ MSA with predominant parkinsonian symptoms (MSA-P): } \\
\hline 1 & $\mathrm{M}$ & 43 & Erectile dysfunction & 1 & 6 & HRS & AS and $G$ & Yes & $3 \cdot 0$ & NT & None \\
\hline 2 & $\mathbf{M}$ & 56 & VCP & 1 & 2 & HRS & AS and $G$ & No & $4 \cdot 0$ & Poor & LD 375, Dep, Brom \\
\hline 3 & $\mathbf{M}$ & 53 & Erectile dysfunction & 2 & 2 & HRS & $\mathrm{AS}$ and $\mathrm{G}, \mathrm{AL}$ & Yes & $2 \cdot 0$ & Poor & None \\
\hline 4 & $\mathbf{M}$ & 66 & Erectile dysfunction & 2 & 20 & HRS, Tr & $\mathrm{AS}$ and $\mathrm{G}, \mathrm{AL}$ & No & $4 \cdot 0$ & Poor & LD 375, Dep, Mem \\
\hline 5 & $\mathrm{~F}$ & 58 & Urinary dysfunction & 4 & 5 & HRS, AC & & Yes & $9 \cdot 0$ & Poor & LD 625, Dep, Lis \\
\hline 6 & $\mathbf{F}$ & 55 & Bradykinesia & 4 & 1 & HRS, AC & No & No & $9 \cdot 5$ & Poor & LD 500, Dep, Lis \\
\hline 7 & $\mathbf{M}$ & 59 & Erectile dysfunction & 7 & 10 & HRS & $\mathrm{AS}$ and $\mathrm{G}, \mathrm{AL}$ & No & $9 \cdot 5$ & Poor & LD 375, Dep, Mem \\
\hline \multicolumn{12}{|c|}{ MSA with predominant cerebellar symptoms (MSA-C): } \\
\hline 8 & M & 59 & Erectile dysfunction & 2 & 4 & No & $\mathrm{AS}$ and $\mathrm{G}, \mathrm{AL}$ & Yes & 3.5 & NT & None \\
\hline 9 & $\mathrm{~F}$ & 49 & Sleep apnoea syndrome & 4 & 6 & No & $\mathrm{AS}$ and $\mathrm{G}, \mathrm{AL}$ & No & $6 \cdot 0$ & NT & None \\
\hline 10 & $\mathbf{M}$ & 55 & $A S$ and $G$ & 7 & 4 & HRS & $\mathrm{AS}$ and $\mathrm{G}, \mathrm{AL}$ & No & $7 \cdot 5$ & NT & None \\
\hline 11 & $\mathbf{M}$ & 51 & $A S$ and $G$ & 5 & 1 & HRS & $\mathrm{AS}$ and $\mathrm{G}, \mathrm{AL}$ & No & $5 \cdot 0$ & NT & None \\
\hline 12 & $\mathbf{M}$ & 44 & $A S$ and $G$ & 6 & 6 & No & $\mathrm{AS}$ and $\mathrm{G}, \mathrm{AL}$ & Yes & $7 \cdot 0$ & NT & None \\
\hline 13 & $\mathbf{M}$ & 40 & Erectile dysfunction & 7 & 8 & No & $\mathrm{AS}$ and $\mathrm{G}, \mathrm{AL}$ & Yes & $8 \cdot 5$ & NT & None \\
\hline 14 & $\mathbf{M}$ & 56 & VCP & 9 & 10 & HRS & $\mathrm{AS}$ and $\mathrm{G}, \mathrm{AL}$ & No & $8 \cdot 0$ & None & None \\
\hline 15 & M & 62 & AS and $G$ & 9 & 5 & HRS & $\mathrm{AS}$ and $\mathrm{G}, \mathrm{AL}$ & No & 8.5 & NT & None \\
\hline 16 & F & 59 & $A S$ and $G$ & 10 & 2 & HRS, Tr & $A S$ and $G, A L$ & Yes & $9 \cdot 0$ & NT & None \\
\hline
\end{tabular}

VCP = vocal cord paralysis; $A S$ and $G=$ ataxia of stance and gait; $M O=$ motor; $A F=$ autonomic failure; HRS = hypokinetic-rigid syndrome; $\mathrm{Tr}=$ tremor; $\mathrm{AC}=$ antecollis; $\mathrm{AL}=$ ataxia of limbs; $\mathrm{NT}=$ not tested; $\mathrm{LD}=$ levodopa + dopa decarboxylase inhibitor $($ daily dose in $\mathrm{mg}) ; \mathrm{Dep}=\mathrm{L}-\mathrm{deprenyl} ; \mathrm{Brom}=$ bromocriptine; $\mathrm{Lis}=$ lisuride, $\mathrm{Mem}=$ memantine. 
Table 2 Details of autonomic failures in personally examined patients

\begin{tabular}{|c|c|c|c|c|c|c|}
\hline Case & $\begin{array}{l}\text { Orthostatic } \\
\text { hypotension }\end{array}$ & $\begin{array}{l}\text { Erectile } \\
\text { dysfunction }\end{array}$ & $\begin{array}{l}\text { Urinary } \\
\text { dysfunction }\end{array}$ & Stridor & Anhidrosis & Diarrhoea \\
\hline $\begin{array}{l}1 \\
2 \\
3 \\
4 \\
5 \\
6 \\
7 \\
8 \\
9 \\
10 \\
11 \\
12 \\
13 \\
14 \\
15 \\
16\end{array}$ & $\begin{array}{l}+ \\
+ \\
+ \\
+ \\
+ \\
+ \\
+ \\
+ \\
+ \\
+ \\
(+) \\
+ \\
+ \\
+ \\
\text { No } \\
++ \\
++ \\
++ \\
+\end{array}$ & $\begin{array}{l}\text { Yes } \\
\text { Yes } \\
\text { Yes } \\
\text { Yes } \\
\star \\
\star \\
\text { Yes } \\
\text { Yes } \\
\star \\
\text { Yes } \\
\text { Yes } \\
\text { Yes } \\
\text { Yes } \\
\text { Yes } \\
\text { Yes }\end{array}$ & $\begin{array}{l}\text { R, I } \\
\text { R, SUC } \\
\text { R, I } \\
\text { R, I } \\
\text { I, SUC } \\
\text { R, I, SUC } \\
\text { R, I, SUC } \\
\text { No } \\
\text { I } \\
\text { R } \\
\text { R, SUC } \\
\text { I, SUC } \\
\text { R, SUC } \\
\text { I , I, SUC } \\
\text { R, I, SUC } \\
\text { I, SUC }\end{array}$ & $\begin{array}{l}\text { NS } \\
\text { T } \\
\text { No } \\
\text { No } \\
\text { NS } \\
\text { T } \\
\text { NS } \\
\text { NS } \\
\text { SAS } \\
\text { NS } \\
\text { NS } \\
\text { NS } \\
\text { NS } \\
\text { T } \\
\text { NS } \\
\text { No }\end{array}$ & $\begin{array}{l}\text { No } \\
\text { No } \\
\text { No } \\
\text { No } \\
\text { No } \\
\text { No } \\
\text { No } \\
\text { No } \\
\text { No } \\
\text { No } \\
+ \\
+ \\
+ \\
+ \\
\text { No } \\
\text { No }\end{array}$ & $\begin{array}{l}+ \\
\text { No } \\
\text { No } \\
\text { No } \\
\text { No } \\
\text { No } \\
\text { No } \\
\text { No } \\
\text { No } \\
+ \\
\text { No } \\
\text { No } \\
\text { No } \\
\text { No } \\
\text { No } \\
\text { No }\end{array}$ \\
\hline
\end{tabular}

Orthostatic hypotension: $(+)=$ orthostatic reduction in systolic blood pressure by $30 \mathrm{mmHg}$ or more, $+=$ symptomatic hypotension with presyncope, $++=$ symptomatic hypotension with syncope; erectile dysfunction: ${ }^{\star}=$ female patient; urinary dysfunction: $\mathrm{R}=$ retention, $\mathrm{I}=$ incontinence, SUC $=$ suprapubic urinary catheter; stridor: $\mathrm{NS}=$ nocturnal stridor, $\mathrm{T}=$ tracheotomy after bilateral vocal cord paralysis, SAS = sleep apnoea syndrome.

tral and obstructive sleep apnoea syndrome was diagnosed. Disturbances of sweating or bowel function were infrequent.

Motor symptoms occurred in various combinations. Ten patients had a combination of parkinsonian and cerebellar features $(63 \%)$, seven without $(44 \%)$ and three with pyramidal signs (19\%). Three patients had cerebellar and pyramidal symptoms without parkinsonism $(19 \%)$. A pure cerebellar syndrome, pure parkinsonism, or a combination of parkinsonism and pyramidal signs occurred in one patient each. In all patients with MSA-P, the first motor symptom was bradykinesia, in all patients with MSA-C it was ataxia of stance and gait. The first motor symptom, whether parkinsonian or cerebellar, determined the clinical type during the entire course of the disease.

In 10 patients (63\%) symptoms of dysautonomia preceded motor signs (table 2), in some of them by more than 10 years. In the remaining six patients in whom the disease started with parkinsonism or ataxia, autonomic symptoms occurred within one to eight years after the onset of motor symptoms. The serial order in which autonomic symptoms occurred was random. Autonomic symptoms were similar in both groups and their duration did not differ significantly between groups (mean (SD) MSA-P 6.6 (6.7) years; MSA-C $5 \cdot 1(2 \cdot 8)$ years). The orthostatic reduction in systolic blood pressure was more pronounced

Figure 2 IBZM binding as detected by SPECT in idiopathic Parkinson's syndrome (IPS), MSA-C, and $M S A-P . B G / F C$ ratio $=$ basal ganglia to frontal cortex ratio of IBZM binding. Solid line represents median of IPS group, dashed line $2 S D$. Froup, dashed line $2 S D$ median value of each group. Shaded symbols represent individual patients with MSA-C wathout parkinsonism $(n=4)$ or patients with $M S A-P$ without ataxia $(n=2)$ respectively.

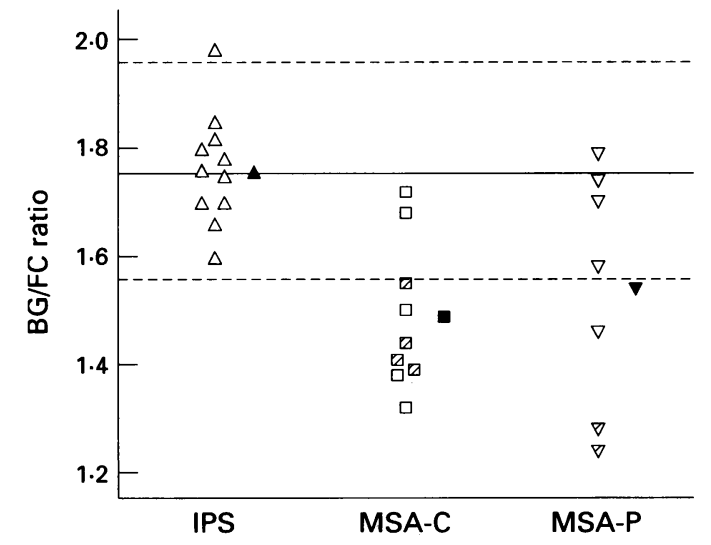

in MSA-P (mean (SD) 50 (24) $\mathrm{mm} \mathrm{Hg}$ ) than in MSA-C (37 (17) $\mathrm{mm} \mathrm{Hg}$; $\mathrm{p}=0.23$ ), but the difference did not reach significance.

\section{IBZM-SPECT}

The $\mathrm{BG} / \mathrm{FC}$ ratio, which serves as a semiquantitative measure of dopamine receptor binding in the striatum, was significantly reduced in MSA (1.50 (0.16) compared with idiopathic Parkinson's syndrome (1.76 $(0 \cdot 10))$. There were no differences between MSA-C $(1.49 \quad(0.14)$ and MSA-P $(1.52$ $(0 \cdot 20))$. Three of the seven patients with MSA-P and seven of the nine patients with MSA-C had BG/FC ratios that were more than 2 SD below the normal range (fig 2). If a threshold of $2.5 \mathrm{SD}$ was chosen, three patients with MSA-P and only six with MSA$C$ were outside the normal range. The BG/FC ratios showed a weak, negative correlation with the Kurtzke score $(r=-0.49 ; \mathrm{p}=0.05)$, but no significant correlation with the scores rating severity of parkinsonism. They were not significantly correlated with the rating scores for putaminal hypointensities.

MRI

All measures of cerebellar and brainstem size (fig 3) were significantly different by analysis of variance between the patient and control groups (cerebellar vermis: $F_{2,36}=58 \cdot 7$, hemispheres: $\quad F_{2,36}=36 \cdot 7$, middle cerebellar peduncle: $\quad F_{2,36}=79 \cdot 6$, basis pontis: $F_{2,36}=$ $57 \cdot 2$, medulla oblongata: $\mathrm{F}_{2,36}=20 \cdot 7 ; \mathrm{p}<$ 0.0001 ), whereas there were no differences in spinal cord size and the estimated degree of cortical atrophy (controls: median 0 , range 0 to 2 ; MSA-P 0.5 , range 0 to 1 ; MSA-C 0 , range 0 to 2). Further analysis of different main effects by Duncan's multiple range test showed that the size of cerebellar and brainstem structures was reduced in the MSA-P and MSA-C groups compared with controls $(p<0.001)$ and that the amount of reduction was greater in MSA-C than in MSA-P (fig 4).

On average, the middle cerebellar peduncle showed the most pronounced size reduction of all infratentorial brain structures (fig 3). Its diameter was reduced by more than $2 \mathrm{SD}$ compared with controls in all but two patients 

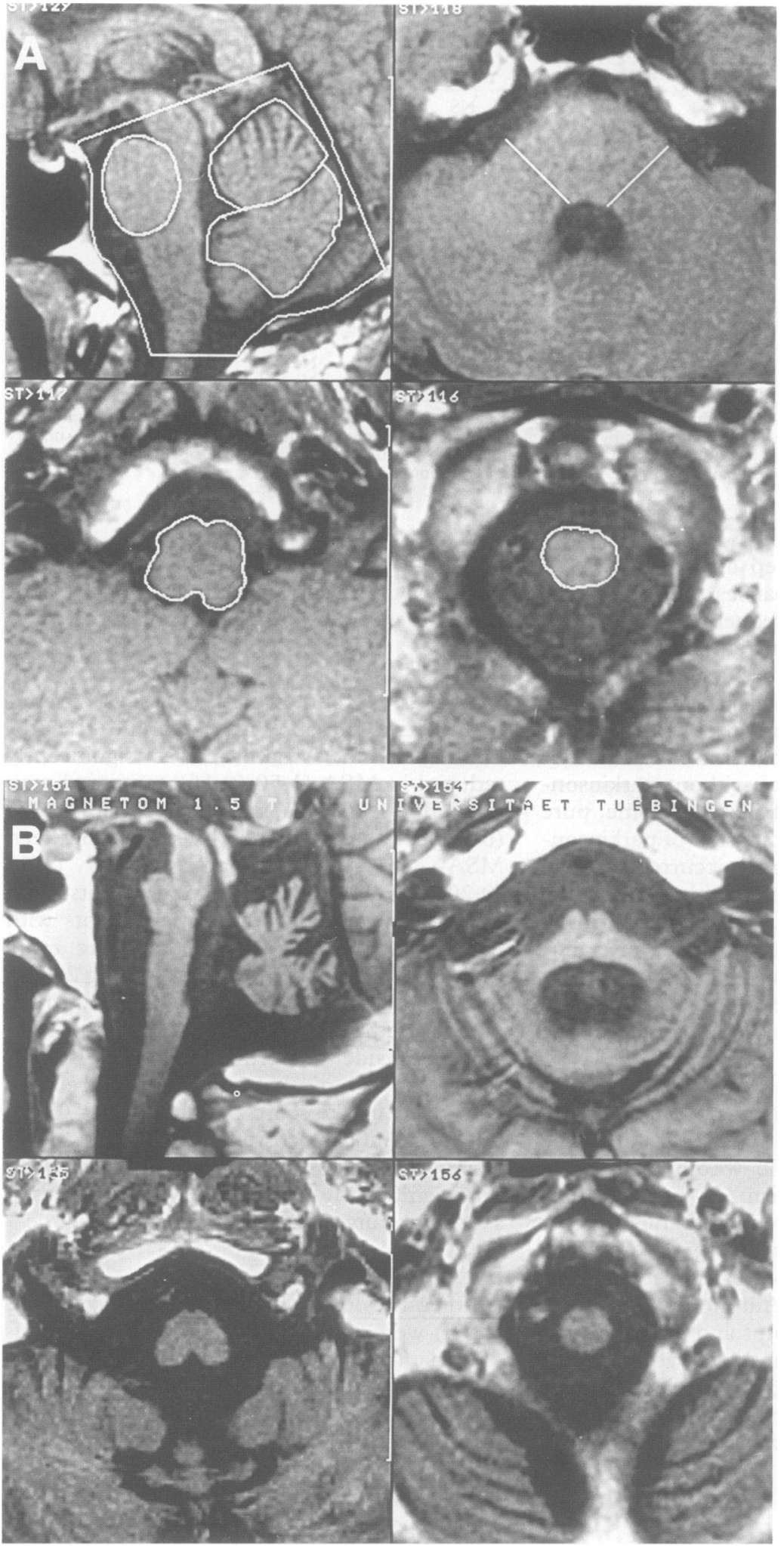

Figure $3 M R I$ (T1 weighted spin echo images $1 \cdot 5 T, T R=600 \mathrm{~ms}, T E=15 \mathrm{~ms}$, slice thickness $=4 \mathrm{~mm}$ ) of infratentorial brain structures showing the posterior fossa in the midsagittal plane (upper left) and axial images at the level of the middle cerebellar peduncles (upper right), inferior olivary complex (lower left) and dens axis (lower right). (A)MRI of a 34 year-old healthy male. The dashed lines outline the size of the posterior fossa, the anterior and posterior vermis, the ventral part of the pons, the maximum diameter of the middle cerebellar peduncles and the area of medulla oblongata and the cervical spinal cord as described in the text. (B) MRI of a 69 year old female patient (case 16) with MSA-C. There is severe atrophy of the anterior and posterior cerebellar vermis, the basis pontis, the middle cerebellar peduncles, and the medulla oblongata.

with MSA. The diameter was negatively correlated with the Kurtzke score $(r=-0.71$; p $=0.004)$; there was no correlation with $\mathrm{BG} / \mathrm{FC}$ ratios of IBZM-SPECT or rating scores for putaminal hypointensities.

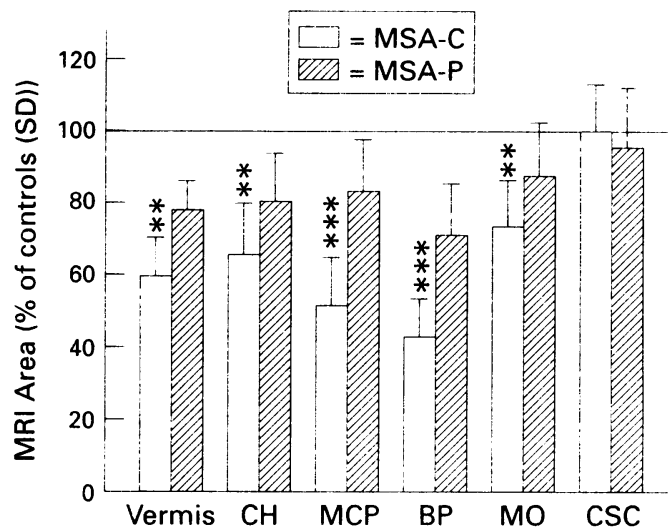

Figure 4 MRI morphology in MSA-C and MSA-P. Sizes of anatomical structures were determined planimetrically and are given as percentages of the respective control values (mean (SD)). Vermis = anterior and posterior cerebellar vermis; $\mathrm{CH}=$ cerebellar hemispheres; $M C P=$ middle cerebellar peduncles; $B P=$ basis pontis; $M O=$ medulla oblongata;

CSC = cervical spinal cord. Comparison of MSA-P and $M S A-C$ was by one way analysis of variance and subsequent Duncan's multiple range test: ${ }^{\star \star} p<0 \cdot 01$; $\star \star \star p<0.001$.

Analysis of the degree $\left(\mathrm{H}_{2,27}=10 \cdot 4\right)$ and area $\left(\mathrm{H}_{2,27}=8.4\right)$ of putaminal hypointensitie identified the presence of significant group effects $(p<0.01)$. The rating scores for the degree of putaminal hypointensities in MSA$C$ (median 2, range 1 to 3 ) and MSA-P (median 2 , range 2 to 3 ) were significantly higher than those of controls (median 0 , range 0 to $2 ; \mathrm{p}<0.05$; figs 5 and $6 \mathrm{~A}$ ). The area of the hypointensities was greater in MSA-P (median 2, range 1 to 3 ) than in MSA-C (median 1.5 , range 1 to 2 ; $\mathrm{p}<0.05$; fig $6 \mathrm{~B}$ ) and both groups differed significantly from controls (median 0 , range 0 to $2 ; p<0.05$ ). No significant differences in the signal intensities of the pallidum, red nucleus, substantia nigra, or dentate nucleus were noted. Putaminal hypointensity exceeding hypointensity of the pallidum has been proposed as an easily accessible indicator of putaminal abnormality. ${ }^{9102526}$ This was true in 12 of 14 patients with MSA, but also in five of 14 control subjects. The rating scores of putaminal hypointensities were not correlated with the Kurtzke scale, the diameter of the middle cerebellar peduncle, or the BG/FC ratio from IBZM-SPECT.

Significant group effects $(\mathrm{p}<0.001)$ for hyperintensities within the pons $\left(\mathrm{H}_{2,27}=16.5\right)$ and middle cerebellar peduncle $\left(\mathrm{H}_{2,27}=21 \cdot 0\right)$ were identified. Subsequent mean contrasts showed that both patient groups had higher scores than controls and that patients with MSA-C had higher scores than patients with MSA-P (figs $6 \mathrm{~B}$ and 7).

\section{Discussion}

MSA is principally a neuropathological entity encompassing striatonigral degeneration, olivopontocerebellar atrophy, and degeneration of the intermediolateral cell columns of the spinal cord. ${ }^{1}$ Clinically patients with MSA present with severe symptomatic autonomic failure and parkinsonian or cerebellar symp- 


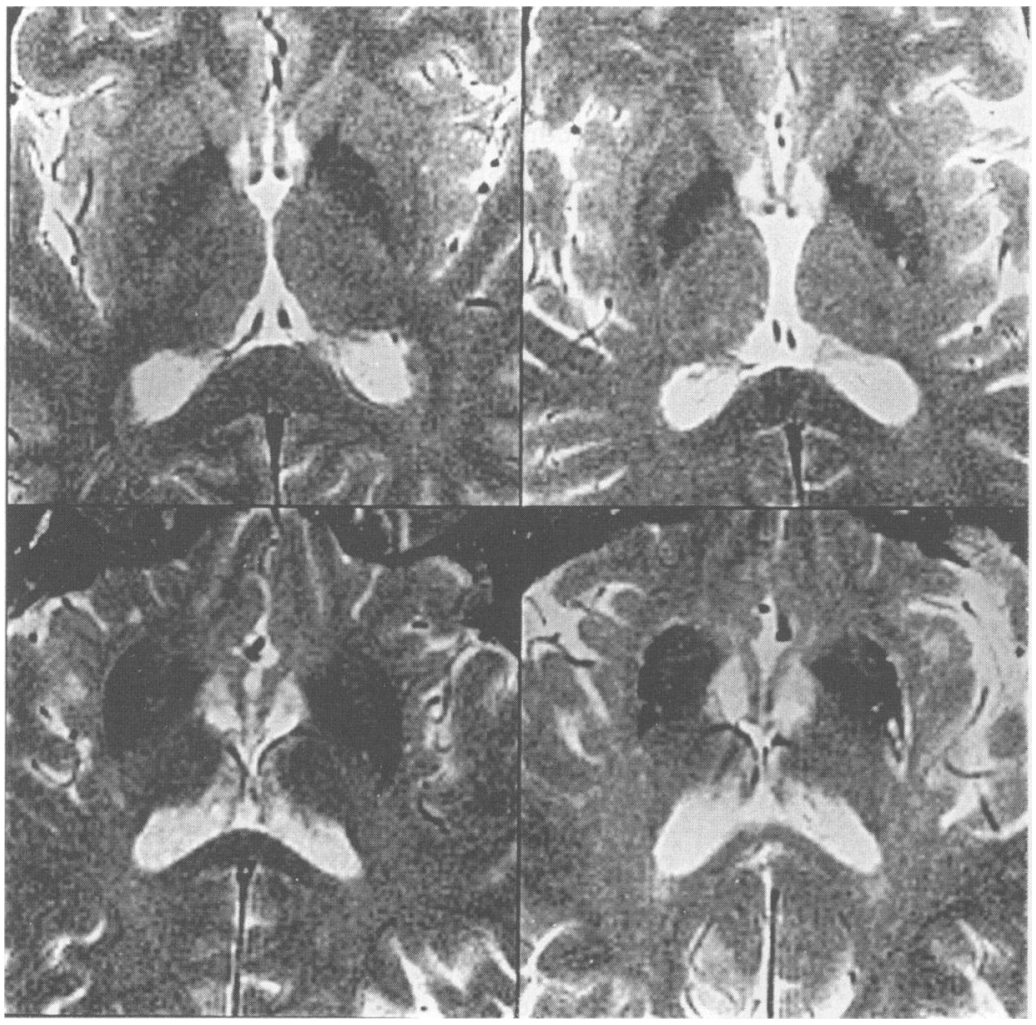

Figure 5 Hypointensities of basal ganglia in MRI (T2 weighted spin echo images 1.5T, $T R=2100 \mathrm{~ms}, T E=45+90 \mathrm{~ms}$, slice thickness $=4 \mathrm{~mm})$. Upper left: 53 year old healthy male. Normal distribution of hypointensities in the basal ganglia. Absence of hypointensity in putamen, normal hypointensity in pallidum (putaminal rating scores: intensity 0, area 0). Upper right: 50 year old male patient with $M S A-C$ (case 12).

Hypointensity at the dorsolateral margin of the putamen (intensity 2, area 1). Lower left: 68 year old male patient with MSA-P (case 4). The strong hypointensity extends through part of the body of putamen (intensity 3; area 2). Lower right: 66 year old male with $M S A-P$ (case 7). Hypointensity extending throughout the entire putamen and intensity exceeding that in globus pallidus (intensity 3; area 3). Note the hyperintensity at the lateral putaminal border.

Figure 6 Severity of putaminal hypointensities (A) and pontine and cerebellar hyperintensities (B) on $T 2$ weighted MRIs. Degree of signal abnormalities was rated independently by two examiners according to the criteria outlined in the methods. Median values are given.

${ }^{\star} p<0.05 ;{ }^{\star \star} p>0.01 v$ controls, Kruskal-Wallis one way analysis of variance by ranks followed by the Mann-Whitney $U$ test.
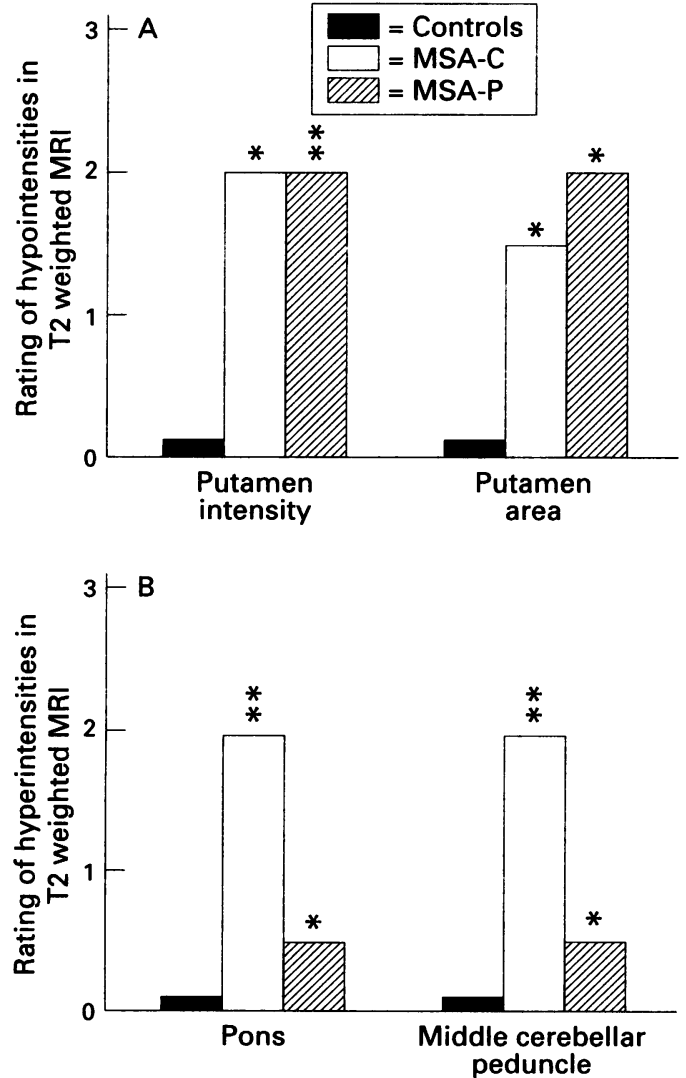

toms. This has led to the clinical distinction between striatonigral degeneration (parkinsonian) and olivopontocerebellar atrophy (cerebellar) type. ${ }^{7} \mathrm{~A}$ major problem in clinical studies of MSA is the uncertainty of the clinical diagnosis. A definite neuropathological diagnosis was made in only two of our patients who died in the course of the study. Although we have used stringent clinical inclusion criteria, some of our patients may have other degenerative diseases. For example, parkinsonism in combination with autonomic failure may also occur in idiopathic Parkinson's disease. ${ }^{27-29}$ On the other hand, patients with cerebellar ataxia or parkinsonism without severe dysautonomia were not included in the present study although they may have unrecognised MSA.

Most recent studies of MSA come from researchers with a special interest in Parkinson's disease. ${ }^{89141530-32}$ This has probably led to an over-representation of patients with parkinsonian MSA in clinical and neuropathological studies of patients with MSA. In the present study, both patients with predominant parkinsonian and predominant cerebellar features were included allowing us to compare their clinical characteristics, prognosis, and results of imaging procedures.

In all patients, the initial motor symptom, whether parkinsonian or cerebellar, determined the clinical type in the entire course of the disease. Nevertheless, with disease progression an overlap of parkinsonian and cerebellar symptoms occurs. Pyramidal symptoms may add to the complex clinical picture in MSA, but are usually not predominant features. By taking a detailed autonomic history we found that autonomic symptoms, in particular erectile dysfunction, preceded the onset of motor symptoms in more than $60 \%$ of our patients, in some of them by more than 10 years. Although it is impossible to prove that these often vague autonomic symptoms occurred as a result of neuronal degeneration in the context of MSA, this finding suggests that MSA may have a presymptomatic phase of several years.

In a review of 213 pathologically established cases of MSA Quinn ${ }^{32}$ reported a median survival after onset of first symptoms of five to six years, whereas we found a longer survival of about nine years in our patients. Possible explanations for the difference between the study of Quinn and the present one is that all of Quinn's patients were diagnosed by pathology, whereas we may have included patients with different disorders, such as idiopathic Parkinson's disease. It is also difficult to pinpoint precisely onset of disease in many patients. More important is the finding that calculated median lifetime and the median time to become wheelchair bound were significantly shorter in MSA-P than MSA-C. These results imply that the prognosis is better in MSA-C than in MSA-P and justify the division into MSA-C and MSA-P at least for clinical and prognostic purposes. The reasons why parkinsonian patients have a worse prognosis are unclear. Possibly, parkin- 


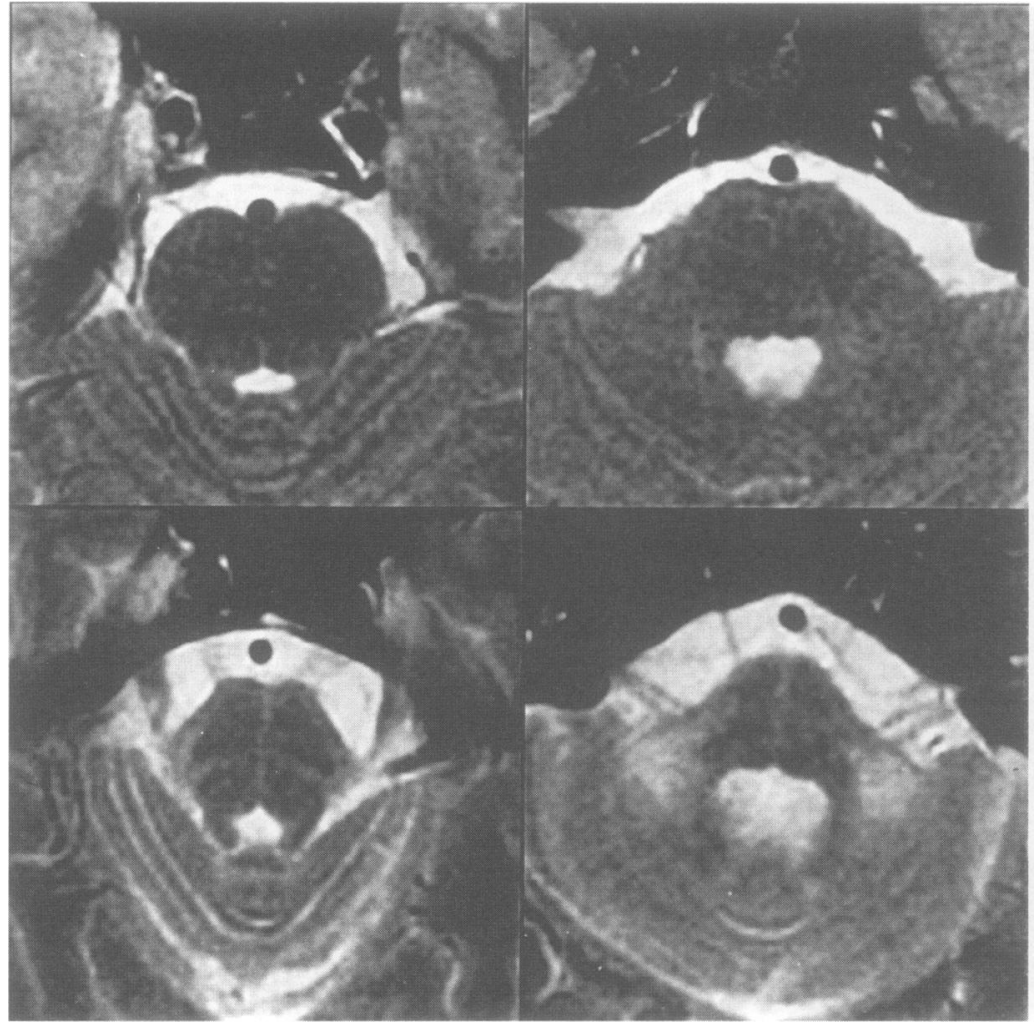

Figure 7 MRI (T2 weighted spin echo images $1 \cdot 5 T, T R=2100 \mathrm{~ms}, T E=45+90 \mathrm{~ms}$, slice thickness $=4 \mathrm{~mm}$ ) of upper pons (left side) and middle cerebellar peduncles (right side). The upper panel shows images of a 53 year old healthy male without signal abnormalities. The lower panel shows hyperintensities of the transverse pontine fibres between tegmentum and the base of pons (left) and in the middle cerebellar peduncles (right) in a 53 year old female patient with MSA-C.

sonian symptomatology and the more severe orthostatic hypotension in patients with MSA-P are the cause of earlier immobility.

Loss of striatal neurons is an integral part of the degenerative process in MSA. ${ }^{1432}$ Dopamine $\mathrm{D}_{2}$ receptors are located on striatal interneurons and on a subset of striatal projection neurons, mainly on those projecting to the external pallidal segment. ${ }^{33}$ Loss of these receptors may thus be taken as an indicator of degeneration of these striatal neurons. Dopamine $\mathrm{D}_{2}$ receptors are imaged by IBZMSPECT. ${ }^{123}$ Pharmacologically, IBZM is a specific dopamine $D_{2}$ antagonist with high affinity for the receptor. Experimental studies in animals show that IBZM binds to areas in the brain with high concentrations of dopamine $\mathrm{D}_{2}$ receptors. ${ }^{23} 34$ Both in animals and in humans, IBZM can be displaced from its binding sites by compounds that are known to specifically interact with dopamine $\mathrm{D}_{2}$ receptors. ${ }^{1234}$ Reduction of striatal IBZM binding should thus reflect a loss of striatal dopamine $\mathrm{D}_{2}$ receptors. By contrast with PET, however, the IBZM-SPECT method yields only semiquantitative data and the sensitivity of the method has not yet been clearly determined. Also, we need to consider whether the medication of our patients interfered with tracer binding. We believe that this is unlikely because medication was stopped 24 hours before SPECT scanning, allowing adequate time for dopaminergic drugs to leave the synaptic cleft. ${ }^{35}{ }^{36} \mathrm{~A}$ further consideration is whether our SPECT results are altered by regional atrophy of the striatum or the prefrontal cortex. Our method of data evaluation leads to an estimation of the relative density of striatal receptors labelled by IBZM (as compared with the prefrontal cortex) rather than to an estimation of total basal ganglia radioactivity. Therefore, shrinkage of the striatum itself would not lead to a reduced BG/FC ratio. On the other hand, cortical atrophy would lead to an understimation of the FC value and an overestimation of the $B G / F C$ ratio. This is improbable in our patients because the MRIs suggest that there is no significant degree of cortical atrophy in patients with MSA. This is in accord with neuropathological findings showing that degeneration of the cerebral cortex is not a typical neuropathological feature of MSA. ${ }^{7}$

Although there was a statistically significant reduction of IBZM binding in patients with MSA compared with controls, only $63 \%$ of our patients had BG/FC ratios that were more than 2 SD below the normal ratio $(56 \%$ below the $2.5 \mathrm{SD}$ threshold). These results closely correspond to those of postmortem receptor binding studies ${ }^{37} 38$ and to a recent PET study of MSA using ${ }^{11} \mathrm{C}$-raclopride to label dopamine receptors. ${ }^{15}$ Although only patients with parkinsonian MSA were included in this study the authors found a modest reduction of $\mathrm{D}_{2}$ receptor binding. We found, surprisingly, that the proportion of patients with pathological IBZM-SPECT and the extent of receptor loss were not different between MSA-C and MSA-P and were sometimes greater in MSA$P$. In particular, individual patients without any parkinsonian symptoms may have a pathological BG/FC ratio in IBZM-SPECT, whereas IBZM binding may be normal in other patients with predominant parkinsonism. These findings indicate that striatal degeneration occurs in patients with MSA independently of their clinical presentation.

It is well known that an extensive loss of striatal neurons, as in the late stages of Huntington's disease, may lead to parkinsonian symptoms. The moderate extent of receptor loss in MSA and the lack of difference between parkinsonian and cerebellar patients suggest that loss of $\mathrm{D}_{2}$ receptors alone does not explain the occurrence of parkinsonian symptoms in MSA. Indeed, Brooks and colleagues ${ }^{14}$ have reported significantly reduced striatal uptake of the positron emitting tracers ${ }^{18} \mathrm{~F}$-dopa and ${ }^{11} \mathrm{C}$-nomifensine, which are markers for dopamine storage capacity and integrity of dopamine uptake sites respectively. Also, a recent PET study reported a moderate loss of $D_{1}$ receptors in the striatum of patients with striatonigral degeneration. ${ }^{39}$ Neuropathological studies show that the pallidal nuclei also undergo severe degeneration in MSA, ${ }^{4}$ which can result in parkinsonian symptoms. ${ }^{40}$ These findings suggest that the development of parkinsonism poorly responsive to levodopa in MSA is due to widespread neuronal degeneration within the basal ganglia rather than to the loss of a single popula- 
tion of neurons as in idiopathic Parkinson's syndrome.

Abnormalities on MRI in MSA include infratentorial atrophy and signal abnormalities on T2 weighted images within the putamen and the pontocerebellar system. The infratentorial atrophy in MSA affects the cerebellar vermis, cerebellar hemispheres, middle cerebellar peduncles, pons, and lower brainstem. This pattern of macroscopic changes closely corresponds with the changes described in neuropathological studies of olivopontocerebellar atrophy. ${ }^{4142}$ With the same technique of in vivo morphometric evaluation we found similar changes in patients with autosomal dominant cerebellar ataxias. ${ }^{43}$ Thus olivopontocerebellar atrophy is not a specific morphological finding in MSA. The infratentorial atrophy in MSA is associated with signal hyperintensities within the pons and middle cerebellar peduncles. These abnormalities, which were first described by Savoiardo et $a l^{2}$ in a heterogeneous group of cerebellar patients, most probably reflect degeneration of pontocerebellar fibres. These fibres originate in the pontine nuclei, cross the midline by taking a transverse course within the pons, and ascend within the contralateral middle cerebellar peduncle. Our finding that infratentorial signal abnormalities are restricted to this fibre tract, and that the middle cerebellar peduncles exhibit the largest size reduction, are strong evidence that the degeneration of the pontocerebellar tract represents the core pathology of olivopontocerebellar atrophy.

Several authors have described the occurrence of putaminal hypointensities in patients with parkinson plus syndromes. ${ }^{8-112526}$ These abnormalities are usually ascribed to accumulation of iron, although this view has been questioned by Brooks and colleagues. ${ }^{44}$ The finding that putaminal hypointensities occur in patients with parkinson plus syndromes with poor response to dopaminergic drugs has led to the suggestion that these changes reflect damage to postsynaptic striatal dopamine receptors. The lack of correlation between putaminal MRI abnormalities and IBZM binding, however, contradicts this view. By contrast, our findings suggest that putaminal iron accumulation and loss of striatal dopamine receptors represent pathological processes that are independent of each other.

The topography of MRI abnormalities was related to the clinical symptomatology. Quantitative and semiquantitative MRI evaluation gave significant differences between the MSA-P and the MSA-C groups. Putaminal hypointensities were more pronounced in patients with predominant parkinsonism, whereas infratentorial atrophy and pontocerebellar hyperintensities were more severe in patients with predominant cerebellar ataxia. Putaminal or infrantentorial MRI changes were, however, not confined to one group of patients. There were patients without obvious parkinsonian signs who had pronounced putaminal hypointensity; on the other hand, infratentorial atrophy or pontocerebellar hyperintensities were present in patients with- out any cerebellar signs. In particular, shrinkage of the middle cerebellar peduncles was found in all cerebellar patients and in all but two parkinsonian patients. In a pathological study, Fearnley and Lees ${ }^{4}$ found pontocerebellar involvement in seven out of 10 cases of striatonigral degeneration. In the remaining three, cell counts of the cerebellar cortex and pontine nuclei were in the low normal range. The present finding of distributed imaging abnormalities in patients with MSA-independent of their clinical presentation-supports the concept that striatonigral degeneration and (sporadic) olivopontocerebellar atrophy are closely interrelated and should be combined under the heading of MSA.

It would be desirable to complement clinical criteria for the diagnosis of MSA by imaging criteria that help to differentiate MSA from other movement disorders. By the neuropathological definition of MSA $^{1732}$ these criteria should include imaging abnormalities within both the basal ganglia and the pontocerebellar system. Unfortunately, imaging procedures to visualise spinal cord involvement in MSA have not yet been developed. The present data show that single criteria, such as pronounced putaminal hypointensity, decreased IBZM binding, or cerebellar atrophy are not sufficient because they lack both specificity and sensitivity. Further studies may show whether a particular combination of imaging criteria will lead to improved diagnosis of MSA.

1 Graham JG, Oppenheimer DR. Orthostatic hypotension and nicotine sensitivity in a case of multiple system atrophy. $\mathcal{F}$ Neurol Neurosurg Psychiatry 1969;32:28-34.

2 Bannister R, Oppenheimer DR. Degenerative disease of the nervous system associated with autonomic failure. Brain 1972;95:457-74.

3 Spokes EG, Bannister R, Oppenheimer DR. Multiple system atrophy with autonomic failure-clinical, histological and neurochemical observations on four cases. logical and neurochemical
$\mathcal{F}$ Neurol Sci 1979;43:59-82.

4 Fearnley JM, Lees AJ. Striatonigral degeneration. A clinicopathological study. Brain 1990;113:1823-42.

5 Gray F, Vincent D, Hauw J. Quantitative study of lateral horn cells in 15 cases of multiple system atrophy. Acta Neuropathol (Berl) 1988;75:513-8.

6 Papp MI, Kahn JE, Lantos PL. Glial cytoplasmatic inclusions in the CNS of patients with multiple system atrophy (striatonigral degeneration, olivopontocerebellar atrophy and Shy-Drager syndrome). I Neurol Sci 1989; 94:79-100.

7 Quinn N. Multiple system atrophy-the nature of the beast. I Neurol Neurosurg Psychiatry 1989;52 (special supplement): 78-89.

8 Drayer BP, Olanow W, Burger P, Johnson GA, Herfkens $\mathbf{R}$, Riederer S. Parkinson plus syndrome: Diagnosis using high field MR imaging of brain iron. Radiology using high field

9 Pastakia B, Polinsky R, Di Chiro G, Simmons JT, Brown R, Wener L. Multiple system atrophy: MR imaging. Radiology 1986;159:499-502.

10 Savoiardo M, Strada L, Girotti F, et al. MR imaging in progressive supranuclear palsy and Shy-Drager syndrome. F Comput Assist Tomogr 1989;13:555-60.

11 Stern MB, Braffman BH, Skolnick BE, Hurtig HI, Grossman RI. Magnetic resonance imaging in Parkinson's disease and parkinsonian syndromes. Neurology 1989;39:1524-6.

12 Brücke T, Podreka I, Angelberger $\mathrm{P}$, et al. Dopamine $\mathrm{D}_{2}$ receptor imaging with SPECT: Studies in different neuropsychiatric disorders. 7 Cereb Blood Flow Metab 1991;11:220-8.

13 Schwarz J, Tatsch K, Arnold G, et al. ${ }^{123}$ I-iodobenzamideSPECT predicts dopaminergic responsiveness in patients with de novo parkinsonism. Neurology 1992;42:556-61.

14 Brooks DJ, Salmon EP, Mathias CJ, et al. The relationship between locomotor disability, autonomic dysfunction, between locomotor disability, autonomic dysfunction,
and the integrity of the striatal dopaminergic system in patients with multiple system atrophy, pure autonomic patients with multiple system atrophy, pure autonomic
failure and Parkinson's disease, studied with PET. Brain 1990;113:1539-52. 
15 Brooks DJ, Ibanez V, Sawle GV, et al. Striatal $\mathrm{D}_{2}$ receptor status in patients with Parkinson's disease, striatonigral degeneration, and progressive supranuclear palsy, measured with "C-raclopride and positron emission tomography. Ann Neurol 1992;31:184-92.

16 Klockgether T, Schroth G, Diener H-C, Dichgans J Idiopathic cerebellar ataxia of late onset: natural history and MRI morphology. $\mathcal{F}$ Neurol Neurosurg Psychiatry and MRI morph

17 Hoehn MM, Yahr MD. Parkinsonism: onset, progression, and mortality. Neurology 1967;17:427-42.

18 Fahn S, Elton RL, Members of the UPDRS Development Committee. Unified Parkinson's Disease Rating Scale. In: Fahn C, Marsden CD, Calne DB, Goldstein M, eds Recent developments in Parkinson's disease. Vol 2. Florham Park, New Jersey: Macmillan Healthcare Information, 1987:153-64

19 Kurtzke JF. Rating neurologic impairment in multiple sclerosis: an expanded disability status scale (EDSS). Neurology 1983;33:1444-52.

20 Schroth G, Naegele T, Klose U, Mann K, Petersen D. Reversible brain shrinkage in abstinent alcoholics, measured by MRI. Neuroradiology 1988;30:385-9.

21 Klose U, Naegele T, Schroth G, Mann K. Volumenbestimmung aus dreidimensionalen MRDatensätzen. In: Nuesslin F, ed. Medizinische Physik. Datensätzen. In: Nuesslin F,
Tübingen: Gulde, 1988:590-4.

22 Savoiardo M, Strada L, Girotti F, et al. Olivopontocerebellar atrophy: $M R$ dignosis and relationship to multisystem atrophy. Radiology 1990;174:693-6.

23 Kung HF, Pan S, Kung MP, Kasliwal R, Reilly J, Alavi A In vitro and in vivo evaluation of [ [123]]IBZM: a potential $\mathrm{CNS} \mathrm{D}_{2}$-dopamine receptor imaging agent. 7 Nucl Med 1989;30:88-92.

24 Kaplan EL, Meier P. Nonparametric estimation from incomplete observations. F Am Stat Assoc 1958;53: 457-81

25 Rutledge JN, Hilal SK, Silver AJ, Defendini R, Fahn S. Study of movement disorders and brain iron by $M R$ American fournal of Neuroradiology 1987;8:397-411.

26 Olanow CW. Magnetic resonance imaging in parkinsonism. Neurol Clin 1992;10:405-20.

27 Goetz CG, Lutge W, Tanner CM. Autonomic dysfunction in Parkinson's disease. Neurology 1986;36:73-5.

28 Koller WC, Vetere-Overfield B, Williamson A, Busenbark $\mathrm{K}$, Nash J, Parrish D. Sexual dysfunction in Parkinson's disease. Clin Neuropharmacol 1990;13:461-3.

29 Sandroni P, Ahlskog JE, Fealey RD, Low PA. Autonomic involvement in extrapyramidal and cerebellar disorders. Clin Auton Res 1991;1:147-55.

30 Hughes AJ, Colosimo C, Kleedorfer B, Daniel SE, Lees AJ. The dopaminergic response in multiple system atrophy. 7 Neurol Neurosurg Psychiatry 1992;55:1009-13.

31 Hughes AJ, Daniel SE, Kilford L, Lees AJ. The accuracy of clinical diagnosis of idiopathic Parkinson's disease: A clinicopathological study. I Neurol Neurosurg Psychiatry. 1992;55:181-4

32 Quinn N. Multiple system atrophy. In: Marsden CD Fahn S, eds. Movement disorders III. London: Fahn S, eds. Movement

33 Harrison MB, Wiley RG, Wooten GF. Changes in $\mathrm{D}_{2}$ but not $D_{1}$ receptor binding in the striatum following selective lesion of striatopallidal neurons. Brain Re:

34 Kung HF, Guo Y-Z, Billings JB, et al. Preparation and biodistribution of $125 \mathrm{I}-\mathrm{IBZM}$ : a potential CNS D-2 dopamine receptor imaging agent. I Nucl Med Biol 1988;15:195-201

35 Drewe J, Mazer N, Abisch E, Krummen K, Keck M Differential effect of food on kinetics of bromocriptine in a modified release capsule and a conventional formulation. Eur $\mathcal{F}$ Clin Pharmacol 1988;35:535 41.

36 Delforge J, Loc'h C, Hantraye P, et al. Kinetic analysis of [76Br]bromolisuride binding to dopamine D2 receptors studied by PET. I Cereb Blood Flow Metal 1991;11: 914-25.

37 Quik M, Spokes E, MacKay A, Bannister R. Alterations in ${ }^{3} \mathrm{H}$-spiperone binding in human caudate nucleus, substantia nigra and frontal cortex in the Shy-Drager synstantia nigra and frontal cortex in the Shy-Drager syn-
drome and Parkinson's disease. $f$ Neurol $S_{c i}$ 1979; an: and $129-37$

38 Cortes R, Camps M, Gueye B, Probst A, Palacios JM. Dopamine receptors in the human brain: autoradiographic distribution of $\mathrm{D} 1$ and $\mathrm{D} 2$ sites in Parkinson syndrome of different etiology. Brain Res 1989;483: $30-8$

39 Shinotoh $\mathrm{H}$, Inoue $\mathrm{O}$, Hirayama $\mathrm{K}$, et al. Dopamine D1 receptors in Parkinson's disease and striatonigral degeneration: a positron emission tomography study. 7 Neuro? Neurosurg Psychiatry 1993;56:467-72.

40 DeLong MR, Georgopoulos AP. Motor functions of the basal ganglia. In: Brookhart JM, Mountcastle VB, eds. Handbook of physiology, section 1 the nervous system, Vol. II motor control, Part 2. Bethesda, Maryland: American Phvsiological Society, 1981:1017-61.

41 Dejerine J, Thomas A. L'atrophie olivo-ponto-cérébelleuse. Nouvelle Iconographie de la Salpétriere 1900;13. belleuse.

42 Greenfield JG. The spino-cerebellar degenerations. Springfield: Charles C Thomas, 1954.

43 Wüllner U, Klockgether T, Petersen D, Naegele T, Dichgans J. Magnetic resonance imaging (MRI) in hereditary and idiopathic ataxia. Neurology 1993;43: 318-25.

44 Brooks DJ, Luthert P, Gadian D, Marsden CD. Does signal-attenuation on high-field T2-weighted MRI of the brain reflect regional cerebral iron deposition? Observations on the relationship between regional cerebral water proton T2-values and iron levels. 7 Neurol Neurosurg Psychiatry 1989;52:108-11. 